\title{
Fe II lifetimes and transition probabilities
}

\author{
R. Schnabel, M. Schultz-Johanning, and M. Kock
}

\author{
Institut für Atom- und Molekülphysik, Abteilung Plasmaphysik, Universität Hannover, Callinstrasse 38, \\ 30167 Hannover, Germany \\ e-mail: roman.schnabel@aei.mpg.de
}

Received 1 September 2003 / Accepted 30 October 2003

\begin{abstract}
Fe II radiative lifetimes were measured applying the time-resolved nonlinear laser-induced fluoresence technique. We investigated 21 levels of up to $47000 \mathrm{~cm}^{-1}$. The uncertainties are typically $2-3 \%$. The lifetimes provide an improved absolute scale to our branching fractions which were measured with a Fourier transform spectrometer and a high-resolution grating spectrometer and which have been published earlier. We report absolute transition probabilities of $140 \mathrm{Fe}$ II lines in the wavelength range $220-780 \mathrm{~nm}$. The overall uncertainties are estimated to be $6 \%$ for the strong and up to $26 \%$ for the weak transitions. The results are compared with recent experimental data from the literature. Our large set of accurate data can be used for a reliability check of theoretical data calculated for iron abundances in astrophysical plasmas.
\end{abstract}

Key words. lifetimes - oscillator strengths - solar iron abundance

\section{Introduction}

We developed an improved apparatus to measure lifetimes below $5 \mathrm{~ns}$ more accurately with the time-resolved laser-induced fluorescence (LIF) technique. The improvements include a more sophisticated evaluation procedure accounting for saturation in the LIF signals. Additionally a linear radio-frequency ion trap (Paul trap) was designed to improve the signal-tonoise ratio in the lifetime measurements. Up to now the most accurate lifetime measurements in Fe II were performed using the fast beam-laser technique (Biémont et al. 1991; Guo et al. 1992). Our improvements to the time-resolved LIF technique now enable a critical comparison of experimental lifetimes derived from both techniques.

A radiative lifetime provides an absolute scale to the branching fractions of that level. The result of this combination is a set of absolute transition probabilities (Huber \& Sandeman 1986; Kock 1996). In Kroll (1985), Kroll \& Kock (1987) and Heise \& Kock (1990) we used lifetimes with high uncertainties of $10 \%$ to calibrate our experimental branching fractions in the Fe II spectrum. These lifetimes were also affected by a systematic error, since they proved to be systematically too large. The recent improvements in lifetime measurements enable us to report a much more reliable set of Fe II transition probabilities of $140 \mathrm{Fe}$ II lines in the wavelength range $220-780 \mathrm{~nm} .13$ of these lines have already been used for a solar iron abundance determination (Schnabel et al. 1999).

Send offprint requests to: $\mathrm{R}$. Schnabel,

e-mail: roman.schnabel@aei.mpg.de

\section{Lifetime measurement}

The time-resolved laser-induced fluorescence (TRLIF) is a standard technique for measuring radiative lifetimes of excited atomic states. The excitation is done with short laser pulses, ideally much shorter than the radiative lifetime under investigation. The LIF signal is recorded over a time scale more than ten times of the radiative lifetime by a fast photo-multiplier in conjunction with a digitizing oscilloscope. Provided that a sufficiently large number of atoms is available, a complete decay curve can be obtained from a single laser pulse. TRLIF therefore is a single-shot method.

In recent papers (Schnabel \& Kock 2000a; Schnabel \& Kock 2000b) we examined in detail the TRLIF method by including for the first time saturation in laser excitation quantitatively. This was done by using a rate equation model for a two-level and for a three-level atom, respectively. We were able to show that a natural lifetime, which is shorter than the laser pulse, can still be measured with an uncertainty of a few percent. Systematic error sources due to saturation or a non-ideal response of the detection system could be eliminated.

The rate equation model is adequate as long as the atoms are not coherently excited. In this regard we have to consider vertical coherences (Rabi oscillations) as well as horizontal coherences (polarization, alignment of sublevels). Overlapping modes in the multimode laser beam produce a broadband excitation and coherence times shorter than the relevant nanosecond time scale of our experiment. Horizontal coherences are produced by the anisotropic excitation of the polarized laser light. In the present experiment we used low saturation 
parameters, i.e. weak laser pumping, although saturation is hard to avoid completely without severe loss in signal-to-noise ratio. With low saturation observation of LIF under the angle of $54.7^{\circ}$ with respect to the axis of laser polarization (magicangle arrangement) ensured a time-resolved LIF signal comparable to isotropic excitation experiments (Hannaford \& Lowe 1983). However, we could not find any anisotropy in the LIF signals when varying the polarization axis of the laser, indicating an internal depolarization mechanism of the trapped ions. This has been confirmed by experiments as discussed by Schnabel \& Kock (2000b).

As a particle source, we used a high-current hollow cathode combined with a linear Paul trap. This apparatus was designed and optimized for pulsed laser spectroscopy on large numbers of metal ions in their ground state or in metastable states (van Lessen et al. 1998).

The Paul trap was operated as an ion guide. Whereas the neutral particles expanded effusively, ionized species were collimated onto the axis due to their charge/mass ratio. The ion trap/guide arrangement consisted of four copper cylindrical rods as guiding electrodes. The thermal population of metastable states inside the discharge was conserved and exploited successfully (Schultz-Johanning et al. 1999).

Two different laser systems were at our disposal. At the Lund Laser Centre (LLC), stimulated Brillouin scattering (SBS) was used to generate short laser pulses below 1 ns (Li et al. 1999b; Norin 1998). Compressed second-harmonic pulses of an injection-seeded Nd:YAG laser were used to pump a dye laser. In the present experiment we obtained linearly polarized pulses of $0.5 \mathrm{~ns}$ duration (FWHM) with a repetition rate of $10 \mathrm{~Hz}$ in the wavelength range 210-270 nm. At our department longer lasting laser pulses of 2 to $3 \mathrm{~ns}$ duration, also linearly polarized and with a repetition rate of $10 \mathrm{~Hz}$, are used. The third harmonic of our Quanta Ray DCR 11-3 Nd:YAG laser pumps a Lambda Physik LPD 3002 dye laser. Overlapping laser modes provided a broadband excitation of the Doppler-broadened ionic lines. We estimated the transition line width of the cooled ions inside the trap to be smaller than $2 \mathrm{GHz}$.

The Fe II ions in the collimated beam were excited resonantly at a distance of $4 \mathrm{~cm}$ or $15 \mathrm{~cm}$, respectively, measured from the small exit aperture. Perpendicular to the laser beam the fluorescence photons were imaged by a lens system onto the photo detector. The crossing of atom beam and laser beam was located in the center of a Helmholtz coil. Magnetic fields of up to $2 \mathrm{mT}$ could be superimposed to check the influence of quantum beats. Also the polarization axis of the laser was varied to investigate anisotropy in the LIF signals. Both effects could not be observed in this experiments on Fe II, although observed previously on different species (cf. Schnabel \& Kock 1997; Schnabel \& Kock 2000a).

Fluorescence photons were recorded time-resolved using a fast photo detector in combination with a fast digitizing oscilloscope (Tektronix TDS $680 \mathrm{~B}$ ). We used two different photo detectors. At LLC a Hamamatsu 1564U microchannel plate (MCP) with a rise-time of 200 ps was at our disposal. Additionally we used a Hamamatsu R2496 photomultiplier with a rise-time of 700 ps. In both cases the response

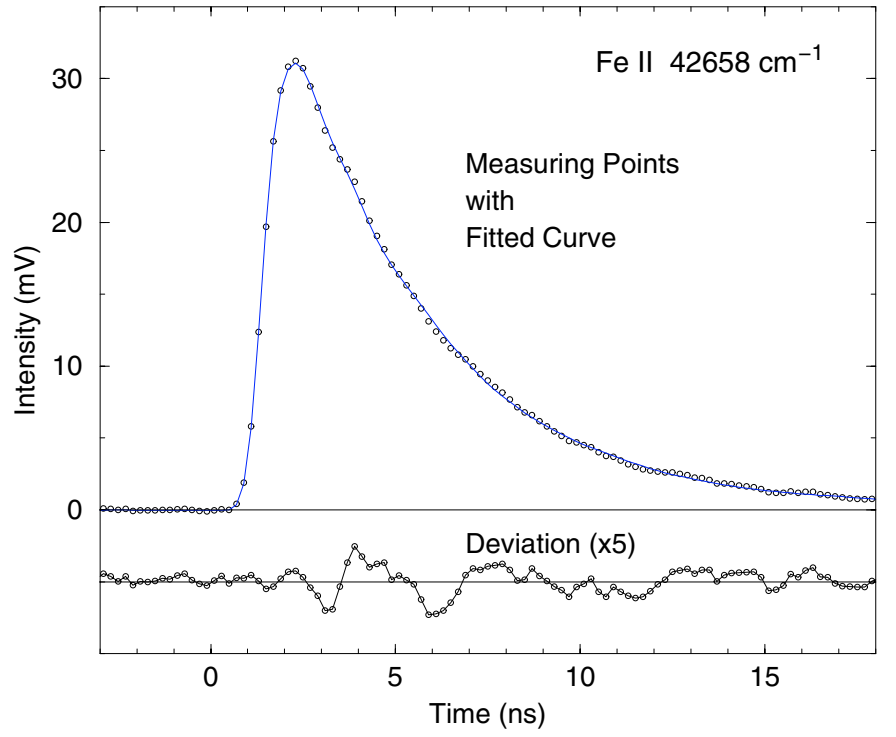

Fig. 1. Time-resolved nonlinear LIF signal during and after a short laser pulse excitation. The circles represent the measurement and the solid line is the fitted theoretical curve. From several of such measurements we determined the lifetime of this Fe II level to $3.71 \mathrm{~ns}$ with a standard deviation of \pm 0.04 ns.

function of the detector system, including signal cable and oscilloscope was measured separately and entered our evaluation procedure Schnabel \& Kock (2000a). Our oscilloscope had an analog bandwidth of $1 \mathrm{GHz}$ and a real-time scanning rate of $2 \times 5$ Gigasamples/s.

The time-resolved nonlinear LIF technique requires recording of the temporal and spatial intensity distributions of the laser pulses. The temporal shape was measured with a fast photo tube (Hamamatsu R1328U-52) simultaneously with the measurement of the LIF signal using the second channel of the oscilloscope. This signal was also used to trigger the fluorescence measurement. The rise-time of that detection system was $(330 \pm 20)$ ps measured with femtosecond laser pulses and was mainly limited by the oscilloscope. The spatial photon distribution of the laser pulses was measured with a $2 / 3$ inch CCIR Standard CCD chip with $756 \times 583$ pixels of $11 \mu \mathrm{m}$ size. For each single laser pulse an 8 -bit histogram of the intensity was recorded. This procedure was fast enough to handle the $10 \mathrm{~Hz}$ repetition rate of our experiment. For the evaluation procedure an averaged 15-bit histogram was calculated from the single shots. The temporal fluorescence was also averaged over the single shots. A typical single lifetime measurement lasted over $100 \mathrm{~s}$ (1000 laser pulses). The measured averaged spatial and temporal laser pulse profiles were used to solve the rate equations for a three-level atom. The result was a temporal process of expected fluorescence intensity which was convoluted with the response function of the detection system and then fitted to the measured signal. For the result we derived the level lifetime which was one of four fitting parameters. For more detailed information we refer to Schnabel \& Kock (2000a) and Schnabel \& Kock (2000b). 
Table 1. Fe II radiative lifetimes.

\begin{tabular}{|c|c|c|c|c|c|c|}
\hline \multirow[t]{2}{*}{ Level } & \multirow{2}{*}{$\begin{array}{c}\text { Energy } E_{2} \\
\left(\mathrm{~cm}^{-1}\right)\end{array}$} & \multirow{2}{*}{\multicolumn{2}{|c|}{$\begin{array}{c}\text { Transitions, this work } \\
\lambda_{\text {exc. }}(\mathrm{nm}) \\
\end{array}$}} & \multicolumn{3}{|c|}{ Lifetimes (ns) } \\
\hline & & & & Ref. [No.] & Ref. [No.] & this work \\
\hline${ }^{6} \mathrm{D}_{9 / 2}^{o}$ & 38458.98 & 259.9396 & 262.5668 & $3.70 \pm 0.06[1]$ & $3.7 \pm 0.2[3]$ & $3.68 \pm 0.07$ \\
\hline${ }^{6} \mathrm{D}_{7 / 2}^{o}$ & 38660.04 & 258.5876 & 261.1874 & $3.68 \pm 0.07[1]$ & $3.75 \pm 0.2$ & $3.67 \pm 0.09$ \\
\hline${ }^{6} \mathrm{D}_{5 / 2}^{o}$ & 38858.96 & 259.8370 & 261.7618 & $3.63 \pm 0.08[1]$ & $3.7 \pm 0.2[3]$ & $3.69 \pm 0.05$ \\
\hline${ }^{6} \mathrm{D}_{3 / 2}^{o}$ & 39013.21 & 260.7088 & 262.0409 & $3.83 \pm 0.10[1]$ & $3.7 \pm 0.2[3]$ & $3.73 \pm 0.07$ \\
\hline${ }^{6} \mathrm{D}_{1 / 2}^{o}$ & 39109.31 & 261.3825 & 262.1670 & $3.76 \pm 0.10[1]$ & $3.8 \pm 0.2[3]$ & $3.68 \pm 0.11$ \\
\hline${ }^{6} \mathrm{~F}_{11 / 2}^{o}$ & 41968.05 & 238.2039 & & $3.19 \pm 0.04[1]$ & $3.2 \pm 0.2[3]$ & ${ }^{*} 3.20 \pm 0.05$ \\
\hline${ }^{6} \mathrm{~F}_{9 / 2}^{o}$ & 42114.82 & 239.5626 & & $3.24 \pm 0.06[1]$ & $3.2 \pm 0.2[3]$ & ${ }^{*} 3.28 \pm 0.04$ \\
\hline${ }^{6} \mathrm{~F}_{7 / 2}^{o}$ & 42237.03 & 238.8630 & 240.4887 & $3.26 \pm 0.10[1]$ & $3.3 \pm 0.2[4]$ & ${ }^{*} 3.25 \pm 0.06$ \\
\hline${ }^{6} \mathrm{~F}_{5 / 2}^{o}$ & 42334.82 & 239.9242 & & $3.33 \pm 0.09[1]$ & $3.3 \pm 0.2[4]$ & $* 3.30 \pm 0.05$ \\
\hline${ }^{6} \mathrm{~F}_{3 / 2}^{o}$ & 42401.30 & 240.6662 & & $3.34 \pm 0.10[1]$ & $3.3 \pm 0.2$ & ${ }^{*} 3.45 \pm 0.12$ \\
\hline${ }^{6} \mathrm{~F}_{1 / 2}^{o}$ & 42439.83 & & & & $3.3 \pm 0.3[4]$ & \\
\hline${ }^{6} \mathrm{P}_{7 / 2}^{o}$ & 42658.22 & 234.3496 & 236.4829 & $3.73 \pm 0.06[2]$ & $3.8 \pm 0.2[3]$ & ${ }^{*} 3.71 \pm 0.04$ \\
\hline${ }^{6} \mathrm{P}_{5 / 2}^{o}$ & 43238.59 & 233.2800 & 234.8303 & $3.79 \pm 0.12[2]$ & $3.7 \pm 0.2$ & ${ }^{*} 3.75 \pm 0.10$ \\
\hline${ }^{6} \mathrm{P}_{3 / 2}^{o}$ & 43620.96 & 232.7397 & & $3.71 \pm 0.12[2]$ & $3.6 \pm 0.2[3]$ & $3.70 \pm 0.12$ \\
\hline${ }^{4} \mathrm{D}_{7 / 2}^{o}$ & 44446.88 & 224.9180 & & $3.02 \pm 0.07[2]$ & $3.1 \pm 0.2[3]$ & $2.97 \pm 0.04$ \\
\hline${ }^{4} \mathrm{D}_{5 / 2}^{o}$ & 44784.76 & 225.1556 & 226.5995 & $3.10 \pm 0.08[2]$ & $3.1 \pm 0.2[3]$ & $2.90 \pm 0.06$ \\
\hline${ }^{4} \mathrm{D}_{3 / 2}^{o}$ & 45044.17 & 226.2688 & & & $3.0 \pm 0.2[3]$ & $2.91 \pm 0.09$ \\
\hline${ }^{4} \mathrm{~F}_{9 / 2}^{o}$ & 44232.51 & 226.0081 & 227.9916 & $3.87 \pm 0.09[2]$ & $3.7 \pm 0.2[3]$ & $3.72 \pm 0.10$ \\
\hline${ }^{4} \mathrm{~F}_{7 / 2}^{o}$ & 44753.80 & 225.3127 & & $3.63 \pm 0.11[2]$ & $3.6 \pm 0.2[3]$ & $3.59 \pm 0.10$ \\
\hline${ }^{4} \mathrm{~F}_{5 / 2}^{o}$ & 45079.88 & 225.0936 & 226.0860 & $3.75 \pm 0.14$ & $3.7 \pm 0.2[3]$ & $3.55 \pm 0.08$ \\
\hline${ }^{4} \mathrm{~F}_{3 / 2}^{o}$ & 45289.80 & & & & $3.7 \pm 0.2[3]$ & \\
\hline${ }^{4} \mathrm{P}_{5 / 2}^{o}$ & 46967.44 & 214.6046 & & $3.43 \pm 0.09[2]$ & & ${ }^{*} 3.27 \pm 0.06$ \\
\hline${ }^{4} \mathrm{P}_{3 / 2}^{o}$ & 47389.78 & 213.9640 & & $3.44 \pm 0.11[2]$ & $3.5 \pm 0.2[5]$ & ${ }^{*} 3.23 \pm 0.09$ \\
\hline
\end{tabular}

[1] Biémont et al. (1991), [2] Guo et al. (1992), [3] Hannaford et al. (1992), [4] Schade et al. (1988),

[5] Li et al. (1999a).

* Lifetime was measured with SBS-compressed laser pulses.

\section{Branching fraction measurement}

In the present paper we use branching fractions from previous publications, the Ph.D. Thesis of Kroll (1985), and the journal papers by Kroll \& Kock (1987) and Heise \& Kock (1990). In all cases branching fractions were determined by means of emission spectroscopy. Additionally Kroll used dispersion (hook) measurements to check the emission measurements to be free from systematic errors. The hook measurements also allowed a consistency check of lifetime data, thereby allowing an optimization of lifetime values which were available from literature. For all emission measurements we used our largescale hollow cathode lamps. The iron spectra were recorded by our $2 \mathrm{~m} \mathrm{McPherson} \mathrm{monochromator} \mathrm{with} \mathrm{a} \mathrm{plane} \mathrm{grating}$ of 2400 lines per $\mathrm{mm}$ and by the $1 \mathrm{~m}$ Fourier-transform spectrometer at Kitt Peak National Observatory in Tucson, Arizona. A detailed description of the experimental procedures used can be found in the three references given above.

\section{Results and discussion}

As already said above, absolute transition probabilities are derived from independent measurements of lifetimes and full sets of branching fractions. Both experimental procedures involve different techniques and are subject to systematic and statistical uncertainties of different size. In the following paragraphs we therefore discuss our lifetime and branching fraction data separately.

Table 1 summarizes the results of our lifetime measurements. Here error bars are given in absolute values and represent a $1 \sigma$ standard deviation derived from up to 20 independent lifetime measurements. Systematic errors have been estimated to be negligible. Comparing our set with accurate literature data we find excellent agreement confirming the absence of systematic error sources in both measuring techniques, the fast beam-laser technique (Biémont et al. 1991; Guo et al. 1992) and the time-resolved non-linear LIF technique used in this 
Table 2. Fe II absolute transition probabilities.

\begin{tabular}{|c|c|c|c|c|c|c|c|}
\hline \multirow{2}{*}{\multicolumn{2}{|c|}{$\begin{array}{l}\text { Upper Level } \\
\qquad E_{2}\left(\mathrm{~cm}^{-1}\right) \\
\tau_{2}(\mathrm{~ns})( \pm \%)\end{array}$}} & \multirow{2}{*}{\multicolumn{2}{|c|}{$\begin{array}{l}\text { Lower Level } \\
\qquad E_{1}\left(\mathrm{~cm}^{-1}\right)\end{array}$}} & \multirow[t]{2}{*}{$\lambda_{\text {air }}(\mathbf{n m})$} & \multicolumn{2}{|c|}{$\mathrm{R}_{21}$} & \multirow{2}{*}{$\begin{array}{l}\mathrm{A}_{21}\left(\mathrm{~s}^{-1}\right) \\
\text { this w. }( \pm \%)\end{array}$} \\
\hline & & & & & Ref.[1] $( \pm \%)$ & this w. $( \pm \%)$ & \\
\hline \multirow[t]{4}{*}{$\mathrm{z}^{6} \mathrm{D}_{9 / 2}^{o}$} & 38458.98 & $a^{6} D_{9 / 2}$ & 0.00 & 259.940 & $8.72 \mathrm{e}-1 \quad(5)$ & $8.65 \mathrm{e}-1 \quad(6)$ & $2.35 e+8 \quad(6)$ \\
\hline & $3.68(2)$ & $a^{6} D_{7 / 2}$ & 384.79 & 262.567 & $1.26 \mathrm{e}-1 \quad(6)$ & $1.34 \mathrm{e}-1 \quad(6)$ & $3.63 e+7 \quad(6)$ \\
\hline & & $\mathrm{a}^{4} \mathrm{~F}_{9 / 2}$ & 1872.57 & 273.245 & & $3.62 \mathrm{e}-4 \quad(8)$ & $9.83 e+4 \quad(8)$ \\
\hline & & $a^{4} D_{7 / 2}$ & 7955.30 & 327.735 & & $1.22 \mathrm{e}-3 \quad(8)$ & $3.31 \mathrm{e}+5 \quad(8)$ \\
\hline \multirow[t]{7}{*}{$\mathrm{z}^{6} \mathrm{D}_{7 / 2}^{o}$} & 38660.04 & $a^{6} D_{9 / 2}$ & 0.00 & 258.588 & $3.17 \mathrm{e}-1 \quad(6)$ & $3.40 \mathrm{e}-1 \quad(6)$ & $9.26 \mathrm{e}+7 \quad(6)$ \\
\hline & $3.67(2)$ & $a^{6} D_{7 / 2}$ & 384.79 & 261.187 & $4.53 \mathrm{e}-1 \quad(5)$ & $4.25 \mathrm{e}-1$ & $1.16 e+8 \quad(6)$ \\
\hline & & $a^{6} D_{5 / 2}$ & 667.68 & 263.132 & $2.29 \mathrm{e}-1 \quad(6)$ & $2.34 \mathrm{e}-1 \quad(6)$ & $6.36 e+7$ \\
\hline & & $a^{4} D_{7 / 2}$ & 7955.30 & 325.589 & & $1.02 \mathrm{e}-3 \quad(8)$ & $2.78 \mathrm{e}+5 \quad(8)$ \\
\hline & & $a^{4} D_{5 / 2}$ & 8391.94 & 330.286 & & $1.02 \mathrm{e}-4(13)$ & $2.78 \mathrm{e}+4(13)$ \\
\hline & & $\mathrm{b}^{4} \mathrm{P}_{5 / 2}$ & 20830.58 & 560.714 & & $1.70 \mathrm{e}-5(16)$ & $4.63 e+3(16)$ \\
\hline & & $a^{6} S_{5 / 2}$ & 23317.63 & 651.608 & & $3.06 \mathrm{e}-5(15)$ & $8.33 e+3(15)$ \\
\hline \multirow[t]{5}{*}{$\mathrm{z}^{6} \mathrm{D}_{5 / 2}^{o}$} & 38858.96 & $a^{6} D_{7 / 2}$ & 384.79 & 259.837 & $5.16 \mathrm{e}-1$ & $5.28 \mathrm{e}-1$ & $1.43 \mathrm{e}+8$ \\
\hline & 3.69 & $a^{6} D_{5 / 2}$ & 667.68 & 261.762 & $1.78 \mathrm{e}-1$ & $1.79 \mathrm{e}-1$ & $4.84 \mathrm{e}+7$ \\
\hline & & $a^{6} D_{3 / 2}$ & 862.61 & 263.105 & $3.05 \mathrm{e}-1$ & $2.92 \mathrm{e}-1 \quad(6)$ & $7.92 \mathrm{e}+7$ \\
\hline & & $a^{4} D_{5 / 2}$ & 8391.94 & 328.129 & & $8.52 \mathrm{e}-4 \quad$ (8) & $2.31 \mathrm{e}+5$ \\
\hline & & $a^{6} S_{5 / 2}$ & 23317.63 & 643.268 & & $3.13 e-5$ (18) & $8.47 e+3(18)$ \\
\hline \multirow[t]{5}{*}{$\mathrm{z}^{6} \mathrm{D}_{3 / 2}^{o}$} & 39013.21 & $a^{6} D_{5 / 2}$ & 667.68 & 260.709 & $6.65 \mathrm{e}-1$ & $6.42 \mathrm{e}-1$ & $1.72 \mathrm{e}+8$ \\
\hline & 3.73 (2) & $a^{6} D_{3 / 2}$ & 862.61 & 262.041 & $1.46 \mathrm{e}-2(14)$ & $1.60 \mathrm{e}-2$ & $4.30 \mathrm{e}+6$ \\
\hline & & $a^{6} D_{1 / 2}$ & 977.05 & 262.829 & $3.19 \mathrm{e}-1 \quad(6)$ & $3.41 \mathrm{e}-1 \quad(6)$ & $9.14 \mathrm{e}+7$ \\
\hline & & $a^{4} D_{3 / 2}$ & 8680.45 & 329.582 & & $7.62 \mathrm{e}-4 \quad(8)$ & $2.04 \mathrm{e}+5 \quad(8)$ \\
\hline & & $a^{6} S_{5 / 2}$ & 23317.63 & 636.946 & & $5.22 \mathrm{e}-5$ & $1.40 \mathrm{e}+4(26)$ \\
\hline \multirow[t]{3}{*}{$\mathrm{z}^{6} \mathrm{D}_{1 / 2}^{o}$} & 39109.31 & $a^{6} D_{3 / 2}$ & 862.61 & 261.383 & $7.93 \mathrm{e}-1 \quad$ & $7.85 \mathrm{e}-1$ & $2.13 e+8$ \\
\hline & $3.68(3)$ & $a^{6} D_{1 / 2}$ & 977.05 & 262.167 & $2.05 \mathrm{e}-1$ & $2.12 \mathrm{e}-1 \quad(6)$ & $5.76 e+7$ \\
\hline & & $a^{4} D_{1 / 2}$ & 8846.77 & 330.346 & & $2.40 \mathrm{e}-4$ (24) & $6.51 \mathrm{e}+4(24)$ \\
\hline $\mathrm{z}^{6} \mathrm{~F}_{11 / 2}^{o}$ & 41968.05 & $a^{6} D_{9 / 2}$ & 0.00 & 238.204 & & & $3.13 \mathrm{e}+8$ \\
\hline \multirow[t]{3}{*}{$\mathrm{z}^{6} \mathrm{~F}_{9 / 2}^{o}$} & 42114.82 & $a^{6} D_{9 / 2}$ & 0.00 & 237.374 & $1.20 \mathrm{e}-1$ & $1.58 \mathrm{e}-1$ & $4.80 \mathrm{e}+7$ \\
\hline & 3.28 (1) & $a^{6} D_{7 / 2}$ & 384.79 & 239.563 & $8.64 \mathrm{e}-1 \quad$ (5) & $8.22 \mathrm{e}-1$ & $2.51 \mathrm{e}+8 \quad$ (6) \\
\hline & & $a^{4} D_{7 / 2}$ & 7955.30 & 292.659 & & $1.68 \mathrm{e}-2(24)$ & $5.12 \mathrm{e}+6(24)$ \\
\hline \multirow[t]{3}{*}{$\mathrm{z}^{6} \mathrm{~F}_{7 / 2}^{o}$} & 42237.03 & $a^{6} D_{7 / 2}$ & 384.79 & 238.863 & $3.14 \mathrm{e}-1$ & $3.71 \mathrm{e}-1$ & $1.14 \mathrm{e}+8$ \\
\hline & & $a^{6} D_{5 / 2}$ & 667.68 & 240.489 & $6.68 \mathrm{e}-1 \quad(5)$ & $6.12 \mathrm{e}-1 \quad(6)$ & $1.88 \mathrm{e}+8$ \\
\hline & & $a^{4} D_{5 / 2}$ & 8391.94 & 295.378 & & $1.69 \mathrm{e}-2$ & $5.20 \mathrm{e}+6(24)$ \\
\hline \multirow[t]{3}{*}{$\mathrm{z}^{6} \mathrm{~F}_{5 / 2}^{o}$} & 42334.82 & $a^{6} D_{5 / 2}$ & 667.68 & 239.924 & $4.58 \mathrm{e}-1 \quad(6)$ & $4.62 \mathrm{e}-1$ & $1.40 \mathrm{e}+8$ \\
\hline & $3.30(2)$ & $a^{6} D_{3 / 2}$ & 862.61 & 241.052 & $5.32 \mathrm{e}-1$ & $4.95 \mathrm{e}-1$ & $1.50 \mathrm{e}+8$ \\
\hline & & $a^{4} D_{7 / 2}$ & 8680.45 & 297.052 & & $8.92 \mathrm{e}-3$ & $2.70 \mathrm{e}+6$ \\
\hline
\end{tabular}

[1] Bergeson et al. (1996).

work. Since we used an ion guide our work was not effected by possible blends and misidentifications due to $\mathrm{Fe} I$ lines. Misidentifications due to Fe II lines were excluded by using more than one excitation wavelength for the upper levels. This was done for more than half of the levels giving us a sufficient absolute wavelength calibration for all the laser dyes used. 
Table 2. continued.

\begin{tabular}{|c|c|c|c|c|c|c|c|}
\hline \multirow{2}{*}{\multicolumn{2}{|c|}{$\begin{array}{l}\text { Upper Level } \\
\qquad E_{2}\left(\mathrm{~cm}^{-1}\right) \\
\tau_{2}(\mathrm{~ns})( \pm \%)\end{array}$}} & \multirow{2}{*}{\multicolumn{2}{|c|}{$\begin{array}{l}\text { Lower Level } \\
\qquad E_{1}\left(\mathrm{~cm}^{-1}\right)\end{array}$}} & \multirow[t]{2}{*}{$\lambda_{\text {air }}(\mathbf{n m})$} & \multicolumn{2}{|c|}{$\mathrm{R}_{21}$} & \multirow{2}{*}{$\begin{array}{l}\mathrm{A}_{21}\left(\mathrm{~s}^{-1}\right) \\
\text { this w. }( \pm \%)\end{array}$} \\
\hline & & & & & Ref.[1] $( \pm \%)$ & this w. $( \pm \%)$ & \\
\hline \multirow[t]{4}{*}{$\mathrm{z}^{6} \mathrm{~F}_{3 / 2}^{o}$} & 42401.30 & $\mathrm{a}^{6} \mathrm{D}_{5 / 2}$ & 667.68 & 239.542 & $8.90 \mathrm{e}-2 \quad(8)$ & $1.08 \mathrm{e}-1(24)$ & $3.13 e+7(24)$ \\
\hline & $3.45(3)$ & $\mathrm{a}^{6} \mathrm{D}_{3 / 2}$ & 862.61 & 240.666 & $5.68 \mathrm{e}-1 \quad(5)$ & $5.23 \mathrm{e}-1$ & $1.52 \mathrm{e}+8$ \\
\hline & & $a^{6} D_{1 / 2}$ & 977.05 & 241.331 & $3.35 \mathrm{e}-1 \quad(6)$ & $3.59 \mathrm{e}-1 \quad(6)$ & $1.04 \mathrm{e}+8$ \\
\hline & & $\mathrm{a}^{4} \mathrm{D}_{7 / 2}$ & 8846.77 & 297.935 & & $5.55 \mathrm{e}-3(24)$ & $1.61 \mathrm{e}+6(24)$ \\
\hline \multirow[t]{2}{*}{$\mathrm{z}^{6} \mathrm{~F}_{1 / 2}^{o}$} & 42439.83 & $a^{6} D_{3 / 2}$ & 862.61 & 240.443 & $1.99 \mathrm{e}-1 \quad(6)$ & $2.27 \mathrm{e}-1 \quad(6)$ & $6.88 \mathrm{e}+7(11)$ \\
\hline & * 3.3 (9) & $a^{6} D_{1 / 2}$ & 977.05 & 241.107 & $7.70 \mathrm{e}-1$ & $7.68 \mathrm{e}-1$ & $2.33 e+8(11)$ \\
\hline \multirow{5}{*}{$\mathrm{z}^{6} \mathrm{P}_{7 / 2}^{o}$} & 42658.22 & $\mathrm{a}^{6} \mathrm{D}_{9 / 2}$ & 0.00 & 234.350 & $6.64 \mathrm{e}-1$ & $6.30 \mathrm{e}-1(11)$ & $1.70 \mathrm{e}+8(11)$ \\
\hline & $3.71(1)$ & $a^{6} D_{7 / 2}$ & 384.79 & 236.483 & $2.20 \mathrm{e}-1 \quad(6)$ & $2.24 \mathrm{e}-1 \quad(11)$ & $6.03 e+7(11)$ \\
\hline & & $a^{6} D_{5 / 2}$ & 667.68 & 238.076 & $1.11 \mathrm{e}-1 \quad(8)$ & $1.19 \mathrm{e}-1(11)$ & $3.21 \mathrm{e}+7(11)$ \\
\hline & & $a^{4} D_{7 / 2}$ & 7955.30 & 288.076 & & $8.20 \mathrm{e}-3(23)$ & $2.21 \mathrm{e}+6(23)$ \\
\hline & & $\mathrm{a}^{6} \mathrm{~S}_{5 / 2}$ & 23317.63 & 516.903 & & $1.56 \mathrm{e}-2(23)$ & $4.22 \mathrm{e}+6(23)$ \\
\hline \multirow[t]{4}{*}{$\mathrm{z}^{6} \mathrm{P}_{3 / 2}^{o}$} & 43620.96 & $a^{6} D_{5 / 2}$ & 667.68 & 232.740 & $2.36 \mathrm{e}-1 \quad(6)$ & $2.18 \mathrm{e}-1(11)$ & $5.89 \mathrm{e}+7(11)$ \\
\hline & $3.70(3)$ & $a^{6} D_{3 / 2}$ & 862.61 & 233.801 & $4.06 \mathrm{e}-1$ & $4.36 \mathrm{e}-1 \quad(11)$ & $1.18 \mathrm{e}+8(11)$ \\
\hline & & $a^{6} D_{1 / 2}$ & 977.05 & 234.428 & $3.44 \mathrm{e}-1 \quad(5)$ & $3.33 \mathrm{e}-1 \quad(11)$ & $8.99 \mathrm{e}+7(11)$ \\
\hline & & $a^{4} D_{7 / 2}$ & 23317.63 & 492.393 & & $1.58 \mathrm{e}-2(23)$ & $4.28 \mathrm{e}+6(23)$ \\
\hline \multirow[t]{7}{*}{$\mathrm{z}^{4} \mathrm{~F}_{9 / 2}^{o}$} & 44232.51 & $a^{6} D_{9 / 2}$ & 0.00 & 226.008 & $1.24 \mathrm{e}-2$ & $1.58 \mathrm{e}-2(24)$ & $4.23 e+6(24)$ \\
\hline & 3.72 & $\mathrm{a}^{6} \mathrm{D}_{7 / 2}$ & 384.79 & 227.992 & $1.74 \mathrm{e}-2$ & & \\
\hline & & $\mathrm{a}^{4} \mathrm{~F}_{9 / 2}$ & 1872.57 & 236.000 & $1.27 \mathrm{e}-1$ & $1.45 \mathrm{e}-1$ & $3.89 e+7$ \\
\hline & & $\mathrm{a}^{4} \mathrm{~F}_{7 / 2}$ & 2430.10 & 239.148 & $1.42 \mathrm{e}-2$ & $1.45 \mathrm{e}-2 \quad(8)$ & $3.89 e+6$ \\
\hline & & $\mathrm{a}^{4} \mathrm{D}_{7 / 2}$ & 7955.30 & 275.574 & $8.24 \mathrm{e}-1$ & $8.09 \mathrm{e}-1$ & $2.17 e+8$ \\
\hline & & $b^{4} F_{9 / 2}$ & 22637.21 & 462.934 & & $6.39 \mathrm{e}-4(24)$ & $1.72 \mathrm{e}+5(24)$ \\
\hline & & $\mathrm{a}^{4} \mathrm{G}_{11 / 2}$ & 25428.78 & 531.662 & & $1.45 \mathrm{e}-3(24)$ & $3.89 \mathrm{e}+5(24)$ \\
\hline \multirow[t]{13}{*}{$\mathrm{z}^{4} \mathrm{~F}_{7 / 2}^{o}$} & 44753.80 & $a^{6} D_{5 / 2}$ & 384.79 & 225.313 & & $1.58 \mathrm{e}-2(13)$ & $4.41 e+6(13)$ \\
\hline & 3.59 (3) & $a^{6} D_{5 / 2}$ & 667.68 & 226.759 & $1.28 \mathrm{e}-2(11)$ & $1.39 \mathrm{e}-2(13)$ & $3.87 e+6(13)$ \\
\hline & & $a^{4} F_{9 / 2}$ & 1872.57 & 233.131 & $1.15 \mathrm{e}-1$ & $1.24 \mathrm{e}-1$ & $3.44 \mathrm{e}+7(13)$ \\
\hline & & $\mathrm{a}^{4} \mathrm{~F}_{7 / 2}$ & 2430.10 & 236.202 & $4.80 \mathrm{e}-2(10)$ & $5.41 \mathrm{e}-2(13)$ & $1.51 \mathrm{e}+7$ \\
\hline & & $a^{4} F_{5 / 2}$ & 2837.95 & 238.501 & $1.21 \mathrm{e}-2(11)$ & $1.39 \mathrm{e}-2(13)$ & $3.87 e+6(13)$ \\
\hline & & $a^{4} D_{7 / 2}$ & 7955.30 & 271.670 & & $3.67 \mathrm{e}-4(24)$ & $1.02 \mathrm{e}+5(24)$ \\
\hline & & $a^{4} D_{7 / 2}$ & 8391.94 & 274.932 & $7.83 e-1 \quad(5)$ & $7.72 \mathrm{e}-1 \quad(13)$ & $2.15 e+8(13)$ \\
\hline & & $\mathrm{a}^{4} \mathrm{P}_{5 / 2}$ & 13474.41 & 319.607 & & $5.79 \mathrm{e}-3(24)$ & $1.61 \mathrm{e}+6(24)$ \\
\hline & & $\mathrm{b}^{4} \mathrm{P}_{5 / 2}$ & 20830.58 & 417.886 & & $6.18 \mathrm{e}-4(24)$ & $1.72 \mathrm{e}+5(24)$ \\
\hline & & $\mathrm{b}^{4} \mathrm{~F}_{7 / 2}$ & 22810.36 & 455.589 & & $8.11 \mathrm{e}-4(24)$ & $2.26 e+5(24)$ \\
\hline & & $\mathrm{b}^{4} \mathrm{~F}_{5 / 2}$ & 22939.36 & 458.284 & & $1.24 \mathrm{e}-4(24)$ & $3.44 \mathrm{e}+4(24)$ \\
\hline & & $a^{4} G_{9 / 2}$ & 25805.33 & 527.600 & & $1.35 \mathrm{e}-3(24)$ & $3.76 e+5(24)$ \\
\hline & & $\mathrm{a}^{4} \mathrm{G}_{7 / 2}$ & 25981.63 & $\mathbf{5 3 2 . 5 5 5}$ & & $2.86 \mathrm{e}-4(24)$ & $7.96 e+4(24)$ \\
\hline
\end{tabular}

[1] Bergeson et al. (1996).

* Lifetime taken from Schade et al. (1988).

In Table 2 we present our branching fractions which are reseparated from the absolute data given in Kroll (1985), Kroll \& Kock (1987), and Heise \& Kock (1990). Possible transitions which were not found in the spectra have been estimated with the data of Kurucz \& Bell (1995) to contribute less than 2\% and have been accounted for in Table 2. We compare our branching fractions with experimental data from Bergeson et al. (1996). In the last row of Table 2 our lifetime data is used for a new absolute scaling of our experimental branching fractions. For two levels, lifetime values have been taken from literature.

In Table 2 all error bars are given in \%. The error bars of our data include statistical and estimated systematic uncertainties. 
Table 2. continued.

\begin{tabular}{|c|c|c|c|c|c|c|c|}
\hline \multirow{2}{*}{\multicolumn{2}{|c|}{$\begin{array}{l}\text { Upper Level } \\
\qquad E_{2}\left(\mathrm{~cm}^{-1}\right) \\
\tau_{2}(\mathrm{~ns})( \pm \%)\end{array}$}} & \multirow{2}{*}{\multicolumn{2}{|c|}{$\begin{array}{l}\text { Lower Level } \\
\qquad E_{1}\left(\mathrm{~cm}^{-1}\right)\end{array}$}} & \multirow[t]{2}{*}{$\lambda_{\text {air }}(\mathbf{n m})$} & \multicolumn{2}{|c|}{$\mathbf{R}_{21}$} & \multirow{2}{*}{$\begin{array}{l}\mathrm{A}_{21}\left(\mathrm{~s}^{-1}\right) \\
\text { this w. }( \pm \%)\end{array}$} \\
\hline & & & & & Ref.[1] $( \pm \%)$ & this w. $( \pm \%)$ & \\
\hline \multirow[t]{15}{*}{$\mathrm{z}^{4} \mathrm{~F}_{5 / 2}^{o}$} & 45079.88 & $a{ }^{6} D_{5 / 2}$ & 667.68 & 225.094 & $1.09 \mathrm{e}-2$ & $1.24 \mathrm{e}-2(13)$ & $3.49 \mathrm{e}+6(13)$ \\
\hline & $3.55(2)$ & $a{ }^{6} D_{3 / 2}$ & 862.61 & 226.086 & $7.40 \mathrm{e}-3$ (12) & $8.40 \mathrm{e}-3$ (13) & $2.37 \mathrm{e}+6(13)$ \\
\hline & & $\mathrm{a}^{4} \mathrm{~F}_{7 / 2}$ & 2430.10 & 234.396 & $1.17 \mathrm{e}-1$ & $1.20 \mathrm{e}-1$ & $3.38 \mathrm{e}+7(13)$ \\
\hline & & $a^{4} F_{5 / 2}$ & 2837.95 & 236.659 & $3.76 \mathrm{e}-2 \quad(8)$ & $4.00 \mathrm{e}-2$ (13) & $1.13 e+7(13)$ \\
\hline & & $a^{4} F_{3 / 2}$ & 3117.46 & 238.236 & $1.20 \mathrm{e}-2(11)$ & $1.16 \mathrm{e}-2(24)$ & $3.27 e+6(24)$ \\
\hline & & $\mathrm{a}^{4} \mathrm{D}_{7 / 2}$ & 7955.30 & 269.283 & $6.10 \mathrm{e}-3$ & & \\
\hline & & $\mathrm{a}^{4} \mathrm{D}_{5 / 2}$ & 8391.94 & 272.488 & $3.61 \mathrm{e}-2 \quad(8)$ & $3.76 \mathrm{e}-2(24)$ & $1.06 \mathrm{e}+7(24)$ \\
\hline & & $\mathrm{a}^{4} \mathrm{D}_{3 / 2}$ & 8680.45 & 274.648 & $7.67 \mathrm{e}-1$ & 7.60e-1 (13) & $2.14 \mathrm{e}+8(13)$ \\
\hline & & $\mathrm{a}^{4} \mathrm{P}_{5 / 2}$ & 13474.41 & 316.309 & & $6.80 \mathrm{e}-4$ (14) & $1.92 \mathrm{e}+5(14)$ \\
\hline & & $\mathrm{a}^{4} \mathrm{P}_{3 / 2}$ & 13673.19 & 318.311 & & $3.48 \mathrm{e}-3$ (13) & $9.80 \mathrm{e}+5(13)$ \\
\hline & & $\mathrm{b}^{4} \mathrm{~F}_{5 / 2}$ & 22939.36 & 451.534 & & $8.40 \mathrm{e}-4$ (24) & $2.37 e+5(24)$ \\
\hline & & $\mathrm{a}^{4} \mathrm{G}_{7 / 2}$ & 25981.63 & 523.463 & & $8.80 \mathrm{e}-4(30)$ & $2.48 \mathrm{e}+5(30)$ \\
\hline & & $\mathrm{a}^{2} \mathrm{~F}_{7 / 2}$ & 27314.92 & 562.750 & & $1.04 \mathrm{e}-5(20)$ & $2.93 e+3(20)$ \\
\hline & & $\mathrm{a}^{2} \mathrm{~F}_{5 / 2}$ & 27620.41 & $\mathbf{5 7 2 . 5 9 6}$ & & $<5.60 \mathrm{e}-6$ & $<1.58 \mathrm{e}+3$ \\
\hline & & $\mathrm{b}^{4} \mathrm{D}_{5 / 2}$ & 31387.95 & 730.156 & & $<8.00 \mathrm{e}-6$ & $<2.25 \mathrm{e}+3$ \\
\hline \multirow[t]{9}{*}{$\mathrm{z}^{4} \mathrm{~F}_{3 / 2}^{o}$} & 45289.80 & $a{ }^{6} D_{3 / 2}$ & 862.61 & 225.018 & & $6.17 \mathrm{e}-3(11)$ & $1.67 \mathrm{e}+6(12)$ \\
\hline & *3.7 (5) & $a^{4} F_{5 / 2}$ & 2837.95 & 235.489 & $9.90 \mathrm{e}-2 \quad$ (7) & $1.07 \mathrm{e}-1$ (11) & $2.89 \mathrm{e}+7(12)$ \\
\hline & & $a^{4} F_{3 / 2}$ & 3117.46 & 237.050 & $5.80 \mathrm{e}-2$ & $7.00 \mathrm{e}-2$ & $1.89 \mathrm{e}+7(12)$ \\
\hline & & $\mathrm{a}^{4} \mathrm{D}_{7 / 2}$ & 8391.94 & 270.938 & & $1.03 \mathrm{e}-3(11)$ & $2.78 \mathrm{e}+5(12)$ \\
\hline & & $\mathrm{a}^{4} \mathrm{D}_{3 / 2}$ & 8680.45 & 273.073 & $1.03 \mathrm{e}-1 \quad$ (7) & $1.07 \mathrm{e}-1$ & $2.89 e+7(12)$ \\
\hline & & $\mathrm{a}^{4} \mathrm{D}_{1 / 2}$ & 8846.77 & 274.320 & $7.28 \mathrm{e}-1$ & 7.00e-1 (11) & $1.89 \mathrm{e}+8(12)$ \\
\hline & & $\mathrm{a}^{4} \mathrm{P}_{1 / 2}$ & 13904.82 & 318.532 & & $1.11 \mathrm{e}-3(11)$ & $3.00 \mathrm{e}+5(12)$ \\
\hline & & $b^{4} F_{3 / 2}$ & 23031.30 & 449.141 & & $7.00 \mathrm{e}-4(23)$ & $1.89 \mathrm{e}+5(24)$ \\
\hline & & $\mathrm{a}^{4} \mathrm{G}_{5 / 2}$ & 26055.42 & 519.758 & & $2.02 \mathrm{e}-3(23)$ & $5.45 \mathrm{e}+5(24)$ \\
\hline \multirow[t]{13}{*}{$\mathrm{z}^{4} \mathrm{D}_{7 / 2}^{o}$} & 44446.88 & $\mathrm{a}^{6} \mathrm{D}_{9 / 2}$ & 0.00 & 224.918 & $9.10 \mathrm{e}-3$ & $1.58 \mathrm{e}-2(24)$ & $5.32 \mathrm{e}+6(24)$ \\
\hline & 2.97 & $\mathrm{a}^{6} \mathrm{D}_{7 / 2}$ & 384.79 & 226.882 & $1.20 \mathrm{e}-3(11)$ & & \\
\hline & & $a^{4} F_{9 / 2}$ & 1872.57 & 234.812 & $1.95 \mathrm{e}-1 \quad$ (7) & $2.11 \mathrm{e}-1$ & $7.09 e+7$ \\
\hline & & $a^{4} F_{7 / 2}$ & 2430.10 & 237.928 & $7.33 \mathrm{e}-2$ & $9.02 \mathrm{e}-2$ & $3.04 \mathrm{e}+7$ \\
\hline & & $\mathrm{a}^{4} \mathrm{~F}_{5 / 2}$ & 2837.95 & 240.260 & $6.50 \mathrm{e}-3(10)$ & $9.40 \mathrm{e}-3$ (24) & $3.17 e+6(24)$ \\
\hline & & $a^{4} D_{7 / 2}$ & 7955.30 & 273.955 & $6.82 \mathrm{e}-1$ & $6.39 \mathrm{e}-1 \quad(6)$ & $2.15 e+8$ \\
\hline & & $\mathrm{a}^{4} \mathrm{P}_{5 / 2}$ & 13474.41 & 322.774 & & $2.63 \mathrm{e}-2(24)$ & $8.86 e+6(24)$ \\
\hline & & $\mathrm{b}^{4} \mathrm{P}_{5 / 2}$ & 20830.58 & 423.317 & & $2.14 \mathrm{e}-3$ (14) & $7.22 \mathrm{e}+5(14)$ \\
\hline & & $b^{4} F_{9 / 2}$ & 22637.21 & 458.384 & & $2.14 \mathrm{e}-3$ (13) & $7.22 \mathrm{e}+5(13)$ \\
\hline & & $\mathrm{b}^{4} \mathrm{~F}_{7 / 2}$ & 22810.36 & 462.052 & & $7.52 \mathrm{e}-5$ (17) & $2.53 e+4(17)$ \\
\hline & & $a^{4} G_{7 / 2}$ & 25981.63 & 541.407 & & $2.78 \mathrm{e}-5(20)$ & $9.37 e+3(20)$ \\
\hline & & b $^{2} \mathrm{H}_{9 / 2}$ & 26352.77 & 552.513 & & $9.40 \mathrm{e}-6$ (24) & $3.17 e+3(24)$ \\
\hline & & $\mathrm{b}^{4} \mathrm{D}_{7 / 2}$ & 31483.18 & 771.172 & & $1.47 \mathrm{e}-4(18)$ & $4.94 \mathrm{e}+4(18)$ \\
\hline
\end{tabular}

[1] Bergeson et al. (1996).

* Lifetime taken from Hannaford et al. (1992).

For the transitions at $430.318 \mathrm{~nm}$ and $523.463 \mathrm{~nm}$ we found different results in our investigations, here we quote the values as given in Heise \& Kock (1990). For a quantitative comparison with data from Bergeson et al. (1996), we quote their error bars including a $5 \%$ calibration uncertainty as given in that paper. For virtually $90 \%$ of all the lines measured, a good common agreement within the mutual error bars has been found. The comparison shows no runaways, however, the sets of Bergeson et al. are far from complete.

Considering now the absolute data in Table 2, we find our new transition probabilities to be increased by about $10 \%$ on average compared with our values published earlier. This corresponds to the new lifetime values applied. The uncertainties in our absolute data are slightly reduced due to the 
Table 2. continued.

\begin{tabular}{|c|c|c|c|c|c|c|c|}
\hline \multirow{2}{*}{\multicolumn{2}{|c|}{$\begin{array}{l}\text { Upper Level } \\
\qquad E_{2}\left(\mathrm{~cm}^{-1}\right) \\
\tau_{2}(\mathrm{~ns})( \pm \%) \\
\end{array}$}} & \multirow{2}{*}{\multicolumn{2}{|c|}{$\begin{array}{l}\text { Lower Level } \\
\qquad E_{1}\left(\mathrm{~cm}^{-1}\right)\end{array}$}} & \multirow[t]{2}{*}{$\lambda_{\text {air }}(\mathbf{n m})$} & \multicolumn{2}{|c|}{$\mathrm{R}_{21}$} & \multirow{2}{*}{$\begin{array}{l}\mathrm{A}_{21}\left(\mathrm{~s}^{-1}\right) \\
\text { this w. }( \pm \%)\end{array}$} \\
\hline & & & & & Ref.[1] $( \pm \%)$ & this w. $( \pm \%)$ & \\
\hline \multirow[t]{15}{*}{$\mathrm{z}^{4} \mathrm{D}_{5 / 2}^{o}$} & 44784.76 & $\mathrm{a}^{4} \mathrm{~F}_{7 / 2}$ & 2430.10 & 236.029 & & $1.81 \mathrm{e}-1 \quad(6)$ & $6.23 \mathrm{e}+7 \quad(6)$ \\
\hline & 2.90 & $\mathrm{a}^{4} \mathrm{~F}_{5 / 2}$ & 2837.95 & 238.325 & & $1.04 \mathrm{e}-1 \quad(6)$ & $3.59 \mathrm{e}+7 \quad(6)$ \\
\hline & & $a^{4} D_{7 / 2}$ & 7955.30 & 271.441 & & $1.65 \mathrm{e}-1 \quad(6)$ & $5.70 \mathrm{e}+7 \quad(6)$ \\
\hline & & $a^{4} D_{5 / 2}$ & 8391.94 & 274.698 & & $4.90 \mathrm{e}-1 \quad(6)$ & $1.69 \mathrm{e}+8 \quad(6)$ \\
\hline & & $a^{4} D_{3 / 2}$ & 8680.45 & 276.894 & & $1.38 \mathrm{e}-2 \quad(8)$ & $4.75 \mathrm{e}+6 \quad(8)$ \\
\hline & & $\mathrm{a}^{4} \mathrm{P}_{5 / 2}$ & 13474.41 & 319.291 & & $3.67 \mathrm{e}-3(24)$ & $1.27 \mathrm{e}+6(24)$ \\
\hline & & $\mathrm{a}^{4} \mathrm{P}_{3 / 2}$ & 13673.19 & 321.331 & & $1.78 \mathrm{e}-2(13)$ & $6.12 \mathrm{e}+6(13)$ \\
\hline & & $\mathrm{b}^{4} \mathrm{P}_{5 / 2}$ & 20830.58 & 417.346 & & $1.29 \mathrm{e}-3(13)$ & $4.43 e+5(13)$ \\
\hline & & $\mathrm{b}^{4} \mathrm{P}_{3 / 2}$ & 21812.06 & 435.177 & & $1.41 \mathrm{e}-3(14)$ & $4.86 \mathrm{e}+5(14)$ \\
\hline & & $\mathrm{b}^{4} \mathrm{~F}_{7 / 2}$ & 22810.36 & 454.947 & & $2.91 \mathrm{e}-3$ (13) & $1.00 \mathrm{e}+6(13)$ \\
\hline & & $\mathrm{b}^{4} \mathrm{~F}_{5 / 2}$ & 22939.36 & 457.634 & & $1.87 \mathrm{e}-4(20)$ & $6.44 \mathrm{e}+4(20)$ \\
\hline & & $\mathrm{a}^{6} \mathrm{~S}_{5 / 2}$ & 23317.63 & 465.698 & & $3.98 \mathrm{e}-5(22)$ & $1.37 \mathrm{e}+4(22)$ \\
\hline & & $\mathrm{a}^{2} \mathrm{~F}_{5 / 2}$ & 27620.41 & 582.442 & & $<1.07 \mathrm{e}-5$ & $<3.68 \mathrm{e}+3$ \\
\hline & & $b^{4} D_{3 / 2}$ & 31364.44 & 744.934 & & $4.88 \mathrm{e}-5$ (25) & $1.68 \mathrm{e}+4(25)$ \\
\hline & & $b^{4} D_{7 / 2}$ & 31483.18 & 751.583 & & $2.35 e-5(26)$ & $8.09 e+3(26)$ \\
\hline \multirow[t]{12}{*}{$\mathrm{z}^{4} \mathrm{D}_{3 / 2}^{o}$} & 45044.17 & $\mathrm{a}^{6} \mathrm{D}_{3 / 2}$ & 862.61 & 226.269 & & $5.76 \mathrm{e}-3 \quad(8)$ & $1.98 \mathrm{e}+6$ \\
\hline & 2.91 & $a^{6} D_{1 / 2}$ & 977.05 & 226.856 & & $1.76 \mathrm{e}-3$ (24) & $6.05 e+5(24)$ \\
\hline & & $\mathrm{a}^{4} \mathrm{~F}_{5 / 2}$ & 2837.95 & 236.860 & $1.81 \mathrm{e}-1 \quad$ (7) & $1.89 \mathrm{e}-1 \quad(6)$ & $6.49 \mathrm{e}+7$ \\
\hline & & $\mathrm{a}^{4} \mathrm{~F}_{3 / 2}$ & 3117.46 & 238.439 & $9.70 \mathrm{e}-2 \quad$ (7) & $1.12 \mathrm{e}-1 \quad(24)$ & $3.85 \mathrm{e}+7(24)$ \\
\hline & & $a^{4} D_{5 / 2}$ & 8391.94 & 272.754 & $2.84 \mathrm{e}-1 \quad(6)$ & $2.72 \mathrm{e}-1 \quad(6)$ & $9.35 \mathrm{e}+7$ \\
\hline & & $a^{4} D_{3 / 2}$ & 8680.45 & 274.918 & $3.64 \mathrm{e}-1 \quad$ (5) & $3.52 \mathrm{e}-1 \quad(6)$ & $1.21 \mathrm{e}+8$ \\
\hline & & $a^{4} D_{1 / 2}$ & 8846.77 & 276.181 & $4.12 \mathrm{e}-2$ & $4.48 \mathrm{e}-2(24)$ & $1.54 \mathrm{e}+7(24)$ \\
\hline & & $\mathrm{a}^{4} \mathrm{P}_{3 / 2}$ & 13673.19 & 318.674 & & $1.12 \mathrm{e}-2 \quad(8)$ & $3.85 \mathrm{e}+6$ \\
\hline & & $\mathrm{a}^{4} \mathrm{P}_{1 / 2}$ & 13904.82 & 321.044 & & $1.06 \mathrm{e}-2$ & $3.63 e+6 \quad(9)$ \\
\hline & & $\mathrm{b}^{4} \mathrm{P}_{3 / 2}$ & 21812.06 & 430.318 & & $6.40 \mathrm{e}-4(26)$ & $2.20 \mathrm{e}+5(26)$ \\
\hline & & $a^{4} D_{7 / 2}$ & 22939.36 & 452.263 & & $2.43 \mathrm{e}-3$ (24) & $8.36 e+5(24)$ \\
\hline & & $a^{4} G_{5 / 2}$ & 26055.42 & 526.481 & & $1.02 \mathrm{e}-4(17)$ & $3.52 \mathrm{e}+4(17)$ \\
\hline \multirow[t]{5}{*}{$\mathrm{z}^{4} \mathrm{P}_{5 / 2}^{o}$} & 46967.44 & $a^{4} D_{7 / 2}$ & 7955.30 & 256.254 & & $5.85 \mathrm{e}-1$ (13) & $1.79 \mathrm{e}+8(13)$ \\
\hline & $3.27(2)$ & $a^{4} D_{5 / 2}$ & 8391.94 & 259.154 & & $1.87 \mathrm{e}-1$ (13) & $5.72 \mathrm{e}+7(13)$ \\
\hline & & $a^{4} D_{3 / 2}$ & 8680.45 & 261.107 & & $2.38 \mathrm{e}-2$ (13) & $7.28 \mathrm{e}+6(13)$ \\
\hline & & $\mathrm{a}^{4} \mathrm{P}_{5 / 2}$ & 13474.41 & 298.483 & & $1.40 \mathrm{e}-1 \quad(24)$ & $4.29 \mathrm{e}+7(24)$ \\
\hline & & $\mathrm{a}^{4} \mathrm{P}_{3 / 2}$ & 13673.19 & 300.265 & & $5.85 \mathrm{e}-2(13)$ & $1.79 \mathrm{e}+7(13)$ \\
\hline \multirow[t]{6}{*}{$\mathrm{z}^{4} \mathrm{P}_{3 / 2}^{o}$} & 47389.78 & $\mathrm{a}^{4} \mathrm{D}_{5 / 2}$ & 8391.94 & 256.348 & & $4.87 \mathrm{e}-1 \quad(6)$ & $1.51 \mathrm{e}+8$ \\
\hline & $3.23(3)$ & $a^{4} D_{3 / 2}$ & 8680.45 & 258.258 & & $2.84 \mathrm{e}-1 \quad(6)$ & $8.80 \mathrm{e}+7$ \\
\hline & & $a^{4} D_{1 / 2}$ & 8846.77 & 259.373 & & $5.28 \mathrm{e}-2 \quad(8)$ & $1.63 \mathrm{e}+7$ \\
\hline & & $\mathrm{a}^{4} \mathrm{P}_{5 / 2}$ & 13474.41 & 294.765 & & $6.50 \mathrm{e}-2 \quad(8)$ & $2.01 \mathrm{e}+7$ \\
\hline & & $\mathrm{a}^{4} \mathrm{P}_{3 / 2}$ & 13673.19 & 296.503 & & $3.04 \mathrm{e}-2 \quad(8)$ & $9.43 e+6 \quad(9)$ \\
\hline & & $a^{4} P_{1 / 2}$ & 13904.82 & 298.555 & & $7.71 \mathrm{e}-2 \quad(8)$ & $2.39 \mathrm{e}+7 \quad(9)$ \\
\hline
\end{tabular}

[1] Bergeson et al. (1996).

higher accuracy of the lifetimes. The measured lifetime for the level $z^{6} \mathrm{~F}_{11 / 2}^{\circ}$ has been directly converted into an absolute probability for the transition at $238.204 \mathrm{~nm}$. Here the branching fraction is almost unity and the contribution from other transitions is not significant within our quoted error bars of $2 \%$.
Although not explicitly given in Table 2, we also compared the absolute transition probabilities. Again good agreement is found for the cases where accurate data are available from literature (Bergeson et al. 1996). Comparing our absolute data with the subset we used for a solar iron abundance determination 
in Schnabel et al. (1999), we find 4 values to be decreased by about $4 \%$ and 4 other values to be increased by $2 \%$. The reasons are slightly changed estimated contributions by unknown lines. Altogether there is no effect on our published solar iron abundance of $\log N(\mathrm{Fe})=7.42 \pm 0.09$.

Our data sets are suitable for a reliability check of theoretical data. We refer to theoretical data that can be found in Kurucz \& Bell (1995), Raassen \& Uylings (1998a) and Raassen \& Uylings (1998b). The consistency with our data is generally quite good although the comparison is not provided in Table 2. In cases where no experimental data is available yet it is generally quite difficult to estimate the quality of theoretical data. The completeness and the accuracy of our data might help theorists to derive such an estimation, taking into account the details of their theoretical codes and the experimental data used for semi-empirical approaches.

In conclusion, we have presented $140 \mathrm{Fe}$ II transition probabilities. Given the high degree of completeness of the sets and the accuracy of the lifetime values used, Table 2 presents one of the most reliable values at present. A substantial improvement in accuracy can only be achieved in the branching fraction data. Although an independent technique to measure strong branches has been demonstrated recently (Schnabel \& Kock 2000b) it remains challenging to reduce systematic and statistical uncertainties in branching fraction measurements.

Acknowledgements. This work was partly supported by the TMR Programme "Access to Large Scale Facilities", Contract No. ERBFMGECT950020 (DG12) and by Deutsche Forschungsgemeinschaft (DFG). We thank the colleagues from Lund Laser Centre (LLC) at Lund University for hospitality and the possibility to use their laser system.

\section{References}

Bergeson, S. D., Mullman, K. L., Wickliffe, M. E., et al. 1996, AJ, 464, 1044

Biémont, E., Baudoux, M., Kurucz, R. L., Ansbacher, W., \& Pinnington, E. H. 1991, A\&A, 249, 539

Guo, B., Ansbacher, W., Pinnington, E. H., Ji, Q., \& Berends, R. W. 1992, Phys. Rev. A, 46, 641

Hannaford, P., \& Lowe, R. M. 1983, Opt. Eng., 22, 532

Hannaford, P., Lowe, R. M., Grevesse, N., \& Noels, A. 1992, A\&A, 259, 301

Heise, C., \& Kock, M. 1990, A\&A, 230, 244

Huber, M. C. E., \& Sandeman, R. S. 1986, Rep. Prog. Phys., 49, 397

Kock, M. 1996, Phys. Scri. T, 65, 43

Kroll, S. 1985, Experimentelle Bestimmung von Übergangswahrscheinlichkeiten des einfach ionisierten Eisens. Ph.D. Thesis, Universität Hannover

Kroll, S., \& Kock, M. 1987, A\&AS, 67, 225

Kurucz, R. L., \& Bell, B. 1995, Atomic spectral line data base. CDROM 23, Harvard Smithsonian Center for Astrophysics, April 15

Li, Z. S., Lundberg, H., Sikström, C. M., \& Johansson, S. 1999a, Eur. Phys. J. D, 6, 9

Li, Z. S., Norin, J., Persson, A., et al. 1999b, Phys. Rev. A, 60, 198

Norin, J. 1998, Development of a laser-pulse compression device based on stimulated brillouin scattering. Master's thesis, Faculty of Technology at Lund University

Raassen, A. J. J., \& Uylings, P. H. M. 1998a, J. Phys. B, 31, 3137

Raassen, A. J. J., \& Uylings, P. H. M. 1998b, A\&A, 340, 300

Schade, W., Mundt, B., \& Helbig, V. 1988, J. Phys. B, 21, 2691

Schnabel, R., \& Kock, M. 1997, Z. Phys. D, 41, 31

Schnabel, R., \& Kock, M. 2000a, Phys. Rev. A, 61, 062506

Schnabel, R., \& Kock, M. 2000b, Phys. Rev. A, 63, 012519

Schnabel, R., Kock, M., \& Holweger, H. 1999, A\&A, 342, 610

Schultz-Johanning, M., Schnabel, R., \& Kock, M. 1999, Eur. Phys. J. D, 5, 341

van Lessen, M., Schnabel, R., \& Kock, M. 1998, J. Phys. B, 31, 1931 